\title{
Knock-Out of Retrovirus Receptor Gene Tva in the Chicken Confers Resistance to Avian Leukosis Virus Subgroups A and $K$ and Affects Cobalamin (Vitamin $B_{12}$ )-Dependent Level of Methylmalonic Acid
}

\author{
Anna Koslová ${ }^{1,+}\left(\mathbb{D}\right.$, Pavel Trefil ${ }^{2}$, Jitka Mucksová ${ }^{2}$, Veronika Krchlíková ${ }^{1}$, Jiří Plachý ${ }^{1}$, Jakub Krijt ${ }^{3}$, \\ Markéta Reinišová ${ }^{1}$, Dana Kučerová ${ }^{1}$, Josef Geryk ${ }^{1}$, Jiří Kalina ${ }^{2} \mathbb{D}$, Filip Šenigl ${ }^{1}$, Daniel Elleder ${ }^{1}{ }^{D}$, \\ Viktor Kožich ${ }^{3}$ (D) and Jiří Hejnar ${ }^{1, *}$
}

1 Institute of Molecular Genetics, Czech Academy of Sciences, Videnska 1083, 14220 Prague, Czech Republic; anna.lounkova@gmail.com (A.K.); veronika.krchlikova@img.cas.cz (V.K.); jiri.plachy@img.cas.cz (J.P.); Marketa.Reinisova@img.cas.cz (M.R.); dana.kucerova@img.cas.cz (D.K.); geryk@img.cas.cz (J.G.); Filip.Senigl@img.cas.cz (F.Š.); daniel.elleder@img.cas.cz (D.E.)

2 BIOPHARM, Research Institute of Biopharmacy and Veterinary Drugs, Pohoři-Chotouň 90, 25449 Jílové u Prahy, Czech Republic; pavel.trefil@bri.cz (P.T.); jitka.mucksova@bri.cz (J.M.); jiri.kalina@bri.cz (J.K.)

check for updates

Citation: Koslová, A.; Trefil, P. Mucksová, J.; Krchlíková, V.; Plachý, J.; Krijt, J.; Reinišová, M.; Kučerová, D.; Geryk, J.; Kalina, J.; et al. Knock-Out of Retrovirus Receptor Gene Tva in the Chicken Confers Resistance to Avian Leukosis Virus Subgroups $\mathrm{A}$ and $\mathrm{K}$ and Affects Cobalamin (Vitamin $\mathrm{B}_{12}$ )-Dependent Level of Methylmalonic Acid. Viruses 2021, 13, 2504. https://doi.org/ $10.3390 / v 13122504$

Academic Editor: Ester Ballana Guix

Received: 10 November 2021 Accepted: 8 December 2021

Published: 14 December 2021

Publisher's Note: MDPI stays neutral with regard to jurisdictional claims in published maps and institutional affiliations.

Copyright: (c) 2021 by the authors. Licensee MDPI, Basel, Switzerland. This article is an open access article distributed under the terms and conditions of the Creative Commons Attribution (CC BY) license (https:// creativecommons.org/licenses/by/ $4.0 /)$
3 Department of Pediatrics and Inherited Metabolic Disorders, Charles University, First Faculty of Medicine and General University Hospital in Prague, 12808 Prague, Czech Republic; jakub.krijt@lf1.cuni.cz (J.K.); Viktor.Kozich@vfn.cz (V.K.)

* Correspondence: hejnar@img.cas.cz

+ Current address: Department of Biomolecular Mechanisms, Max Planck Institute for Medical Research, 69120 Heidelberg, Germany.

Abstract: The chicken Tva cell surface protein, a member of the low-density lipoprotein receptor family, has been identified as an entry receptor for avian leukosis virus of classic subgroup A and newly emerging subgroup $\mathrm{K}$. Because both viruses represent an important concern for the poultry industry, we introduced a frame-shifting deletion into the chicken tva locus with the aim of knockingout Tva expression and creating a virus-resistant chicken line. The tva knock-out was prepared by CRISPR/Cas9 gene editing in chicken primordial germ cells and orthotopic transplantation of edited cells into the testes of sterilized recipient roosters. The resulting tva $-/-$ chickens tested fully resistant to avian leukosis virus subgroups $A$ and $K$, both in in vitro and in vivo assays, in contrast to their susceptible $t v a+/+$ and $t v a+/-$ siblings. We also found a specific disorder of the cobalamin/vitamin $\mathrm{B}_{12}$ metabolism in the tva knock-out chickens, which is in accordance with the recently recognized physiological function of Tva as a receptor for cobalamin in complex with transcobalamin transporter. Last but not least, we bring a new example of the de novo resistance created by CRISPR/Cas9 editing of pathogen dependence genes in farm animals and, furthermore, a new example of gene editing in chicken.

Keywords: avian leukosis virus subgroups $\mathrm{A} / \mathrm{K}$; tva; gene editing in chicken; vitamin $\mathrm{B}_{12} /$ cobalamin

\section{Introduction}

The retrovirus replication cycle starts with the attachment of a retrovirus particle to the host cell and reconformation of retroviral envelope (Env) glycoproteins. Reconformed Env molecules expose hydrophobic fusion peptides and insert them into the host cell cytoplasmic membrane. The process continues with fusion of both viral and host cell membranes and results in virus uptake, uncoating, reverse transcription, and trafficking toward the cell nucleus. Whatever details of the virus entry may differ between retrovirus genera, the Env reconformation is always primed by a lock-key interaction with a highly specific host cell receptor. Display of the proper receptor on the cell surface determines the 
host range and tissue specificity of a given retrovirus, and vice versa, the absence of receptor molecules means that a given cell or animal species is refractory to the virus infection.

Avian leukosis virus (ALV) exemplifies the principles of receptor-dependent virus entry. In total, seven ALV subgroups (A through E, J, and K) have been described in domestic chickens [1]. Each subgroup is highly specific as to the envelope glycoproteins and receptor usage. We now recognize four receptor proteins that are necessary for the host cell infection by ALV subgroups: Tva protein related to the low-density lipoprotein receptors (LDLR) [2,3], Tvb protein belonging to the tumor necrosis factor receptor family [4-6], Tvc protein similar to mammalian butyrophilins [7], and Tvj, which was identified as a $\mathrm{Na}^{+} / \mathrm{H}^{+}$exchanger 1 [8]. For Tva, Tvb, and Tvc receptors, multiple mutated variants were identified that do not bind the ALV Envs because of frame-shift deletions or protein-fold changing amino acid substitutions, mostly cysteine or tryptophan [9-11]. Segregation of such resistant ALV receptor alleles has been observed, and susceptible/resistant breeds or inbred lines of chicken have been characterized.

ALV subgroup A and newly emerging subgroup K share the same Tva receptor [12]. Amino acid residues critical for ALV-A Env binding have been identified within the single cysteine-rich domain delineated between residues C11 and C50 [13-15]. No similar studies exist for ALV-K. In contrast to the $t v a^{s}$ allele encoding, the standard Tva receptor sensitive to ALV-A/K, there are also receptor-defective alleles $t v a^{r}$ (substitution C40W) and $t v a^{r 2}$ (frame-shifting four-nucleotide insertion) that result in resistant birds when present in homozygous state in inbred chicken lines $\mathrm{C}$ and $7_{2}$, respectively [9]. In addition to the full resistance conferred by the $t v a^{r}$ and $t v a^{r 2}$ alleles, we described strongly decreased susceptibility to ALV-A in one semi-inbred chicken line and Indian red jungle fowl (Gallus $g$. murghi), which is the result of intronic deletions within the tva gene and inefficient splicing of the tva transcript [16]. Defective splicing due to the deletion of branch-point signal turned out to be also prevalent in outbred chickens [17]. Thus, natural polymorphisms within the twa gene could be used for breeding toward the resistance to ALV-A and ALV-K, two important concerns of the poultry industry. However, introducing an ALV-A-resistant allele into commercial chicken lines is a lengthy process, and a biotechnological shortcut such as CRISPR/Cas9-mediated knock-out or gene editing of tva gene might be much easier. Quite recently, such genetic knock-out has been demonstrated in cultured chicken cells by introduction of frame-shift mutations into the tva gene coding sequence $[18,19]$.

The physiological function of Tva has remained at the level of speculation up to now. Its orthology with the mammalian CD320 (originally denoted 8D6A) gene [9] provided a clue when CD320 was identified as a cellular receptor for the uptake of cobalamin (cbl, vitamin $B_{12}$ )/transcobalamin II complex in humans [20]. This function of Tva has recently been confirmed by showing internalization of labeled cbl in chicken cells: the tva knock-out decreases cbl uptake ten times, whereas overexpression of tva displays a tenfold increase in $\mathrm{cbl}$ uptake [21]. Furthermore, ALV-A infection interferes with cbl uptake. Because cbl serves as an important metabolic regulator, and cbl deficiency manifests by apparent pathology in humans (reviewed in [22]), animal models are highly needed for comparative studies. A mouse model is already available [23] and demonstrates shifted values of intermediates in cbl-dependent metabolic cycles [24].

In the present study, we introduced a frame-shift mutation into the tva gene in chicken primordial germ cells (PGC) and obtained the in vivo genetic knock-out, tva $\mathrm{KO}$, using the previously elaborated technique of orthotopic male germ line transplantation $[25,26]$. We employed a series of in vitro and in vivo techniques to show full resistance to infection with viruses of both ALV-A and ALV-K subgroup specificity. We detected increased levels of methylmalonic acid (MMA) in the sera of tva - / - chickens, which is consistent with MMA accumulation as a result of cbl depletion and inefficient conversion of methylmalonyl-CoA to succinyl-CoA. Thus, we demonstrated that the resistance to ALV-A and ALV-K could be engineered biotechnologically to establish an alternative model of mild cbl deficiency. 


\section{Materials and Methods}

\subsection{Ethical Statement}

In this work, we conducted all experiments and procedures in accordance with the Czech legislation for animal handling and welfare. The Animal Commodities Department of the Ministry of Agriculture of the Czech Republic approved all animal experiments described in this study (approval no. 65823/2019-MZE-18134). Anesthesis of recipient roosters in the procedure of orthotopic transplantation was performed by intramuscular injection of $15 \mathrm{mg} / \mathrm{kg}$ ketamine (Narkamon, Bioveta, Czech Republic) and $4 \mathrm{mg} / \mathrm{kg}$ xylazine (Rometar, Bioveta, Czech Republic).

\subsection{Experimental Chickens}

Hybrid chicken embryos $\left(\sigma^{\top} \mathrm{CC} 21 \times \mathrm{L} 15 \times\right.$ qoutbred $\left.\mathrm{SH}\right)$ were used as a source of PGCs, and hybrid recipient roosters $\left(\sigma^{\wedge} \mathrm{CC} 21 \times\right.$ \% 15$)$ were used for orthotopic PGC transplantation. SH hens were used for insemination. Inbred lines CC21, L15, and outbred population $\mathrm{SH}$ used for production of experimental chickens were maintained at the Institute of Molecular Genetics, Czech Academy of Sciences, Prague, Czech Republic [27]. Standard husbandry conditions applied in this work included $16 \mathrm{~h}$ light $/ 8 \mathrm{~h}$ dark cycle, food/water provided ad libitum, and housing the birds in either deep litter individual cages (recipient roosters) or battery cages (inseminated hens). The chickens experimentally infected with replication-competent or tumor-inducing viruses were housed together and kept separate from the breeding birds. The laid eggs were incubated in a forced air incubator (BIOS MIDI).

\subsection{PGC Cultivation and Tva Editing}

We derived PGCs from the blood aspirations of WL/B chicken embryos incubated for $2.5 \mathrm{~d}$, which corresponds to the Hamburger and Hamilton stage 16, using the procedures described in [28]. Briefly, we transferred $5 \mu \mathrm{L}$ of blood from the dorsal aorta in a 48-well plate with $150 \mu \mathrm{L}$ of Avian KO-DMEM (Thermo Fisher Scientific, Waltham, MA, USA) supplemented with growth factors, as described previously [29]. We expanded the PGCs for 2 to 3 weeks up to $10^{5}$ cells, isolated DNA from aliquots of PGCs using the PureGene kit (Qiagen, Hilden, Germany), and determined the sex by detection of the W chromosome. Oligonucleotide primers and cycling conditions used for $\mathrm{W}$ detection are described in [30]. One proliferative male PGC line was selected for the tva knock-out at the age of $52 \mathrm{~d}$ in culture. We used the PX458 vector pSpCas9BB-2A-GFP; AddGene no. 48138 [31] and prepared a CRISPR/Cas9 construct by cloning the tva-specific guide RNA sequence into the sg scaffold. The pX458-TVA construct [18] was designed to target the second exon of the tva gene (Figure 1A). PGCs $\left(2.5 \times 10^{5}\right)$ were suspended in Nucleofector Solution V (Lonza) and mixed with $10 \mu \mathrm{g}$ of pX458-TVA in total volume of $100 \mu \mathrm{L}$. Nucleofection was performed with the AMAXA nucleofector (Lonza) using the A-27 program. GFP-positive cells with the highest fluorescence intensity (top 5\%) were single-cell-sorted $3 \mathrm{~d}$ post nucleofection and expanded for two weeks. The resulting clones were genotyped by amplification and sequencing the target region of the tva gene; oligonucleotide primers and cycling conditions used for tva characterization are described in [18].

\subsection{Preparation of Sterile Recipient Roosters and PGC Transplantation}

We used orthotopic PGC transplantation into roosters with suppressed endogenous spermatogenesis in order to obtain F1 offspring with the edited tva gene. The procedures of rooster irradiation and PGC transplantation were described previously $[26,28,30]$. Briefly, hybrids of inbred lines CC21I $\times$ L15 at the age of 8 months were irradiated with five doses of 8 Gy over two weeks using the Terabalt radiation unit (UJP Prague) with ${ }^{60} \mathrm{Co}$ as a source of gamma rays. Subsequent decline of endogenous spermatogenesis was monitored in semen samples collected by dorsoabdominal massage. Azoospermic roosters were anesthetized and bilaterally injected through tunica albuginea with a dose of $1.9 \times 10^{6}$ PGCs, clone 2 in $250 \mu \mathrm{L}$ of culture medium. This transplanted PGCs clone spent 118 days in culture after 
the chicken embryo blood aspiration. We did not encounter any mortality or side effects associated with irradiation, anesthesia, or transplantation surgery.

A

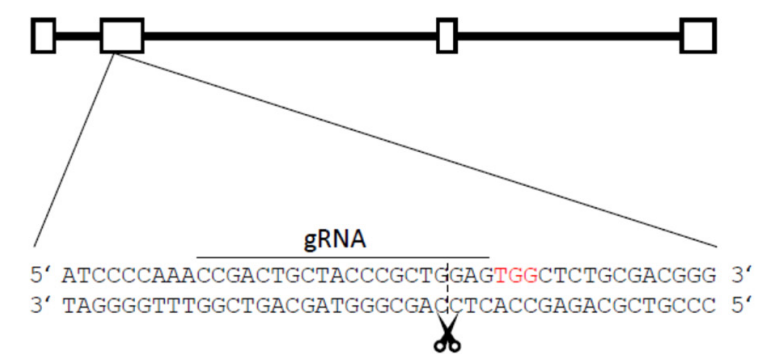

B

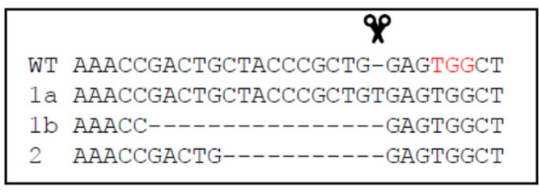

C
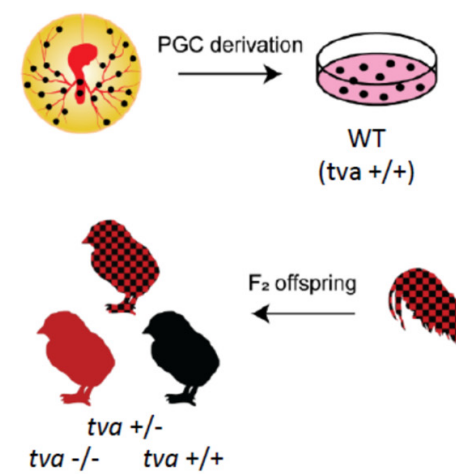
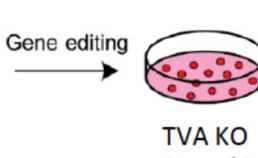
(tva $-/-)$

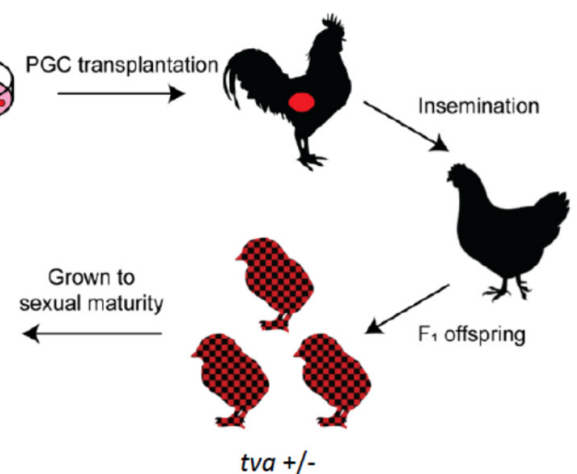

Figure 1. Generation of the tva gene knock-out in chicken PGCs and in vivo in the chicken. (A) Schematic representation of the $t v a$ coding sequence with exons and introns (top) and the design of gRNA used for CRISPR/Cas9 targeting of the tva gene (bottom). The target region of the tva gene is shown with the cleavage site (scissors), PAM sequence (in red), and gRNA sequence depicted with the line above. (B) Alignment of nucleotide sequences of the target region in wt and two PGC clones used for further work with out-of-frame deletions. (C) Schematic summary of the workflow and timeline during generation of $t v a-/-$ chickens.

\subsection{Breeding of Tva KO Chickens}

Appearance of PGC-derived spermatogenesis was monitored in semen samples collected by dorsoabdominal massage the third week after transplantation. The threshold for intramagnal insemination was the semen concentration of $10^{4}$ sperms per $\mathrm{mL}$, and 0.1 to $0.4 \mathrm{~mL}$ of undiluted semen was used as the insemination dose. After successful intramagnal insemination, we also applied intravaginal insemination of SH line hens. The tva + / - genotype of $\mathrm{F}_{1}$ offspring was tested by PCR amplification as described above, grown for at least five months up to sexual maturity and mated in order to obtain $\mathrm{F}_{2}$ progeny segregating $t v a+/+, t v a+/-$, and $t v a-/-$ genotypes.

\subsection{Off-Target Site Selection and Analysis}

We used the CRISPOR tool (http:/ / crispor.tefor.net/ (accessed on 12 September 2019)) for screening the NCBI chicken genome and selection of the most probable off-target sites. Candidate loci with the highest CFD scores, which contained at least 10 consecutive nucleotides matched with the 20-bp tva gRNA sequence and the NGG protospacer adjacent motif, were chosen for further analysis. PCR amplification primers were designed for each of the candidate off-target sites (Supplementary Figure S1), and the respective off-target regions were amplified using $100 \mathrm{ng}$ of genomic DNA of the tva-/- PGC clone 2 and ejaculate of the recipient rooster No. 16. The PCR amplification was carried out under the following conditions: $3 \mathrm{~min}$ at $98^{\circ} \mathrm{C}, 40$ cycles of $10 \mathrm{~s}$ at $98^{\circ} \mathrm{C}, 30 \mathrm{~s}$ at $62^{\circ} \mathrm{C}, 30 \mathrm{~s}$ at $72{ }^{\circ} \mathrm{C}$, and final amplification for $5 \mathrm{~min}$ at $72{ }^{\circ} \mathrm{C}$. Sequence analysis of the resulting PCR products was performed, and the sequences were aligned to the respective NCBI tva sequence. 


\subsection{In Vivo Infection of Chickens and Viremia Analysis}

tva $+/+, t v a+/-$, and $t v a-/-$ chickens at the age of 23 to 39 days were infected by i.v. injection of $10^{6}$ IU of either RCASBP(A)GFP or RCASBP(K)GFP [12,16,32]. Nine and 13 d p.i., blood samples were collected, and sera were prepared. QIAamp Viral RNA Mini Kit (Qiagen) was used for virus RNA isolation from the chicken sera, according to the manufacturer's protocol. Five microliters of prepared RNA was reversely transcribed with Protoscript II Reverse Transcriptase (NEB) and 3'CDS primer (5'AAGCAGTGGTATCAACG CAGAGTACT $_{30} \mathrm{VN}^{\prime}$ ). An amount of $1.5 \mu \mathrm{L}$ of cDNA was then used for quantitative PCR with the MESA GREEN qPCR MasterMix Plus (Eurogentec) and primers for RCAS-A-env (Forward 5'GGGATGCGTAGGCTTCAGA3', Reverse 5'AAAATCTGTAGCCATATGCACCG3') or RCAS-K-env (Forward 5'GCCCCCGGAGCATTGACAA3' , Reverse 5'GGACCTGTCTGTG AACAATTATATAGC $3^{\prime}$ ). To generate the standard curve for absolute quantification of gene expression, we used serial dilution of RCASBP-A and RCASBP-K plasmids. The samples were run in a Bio-Rad CFX96 $6^{\mathrm{TM}}$ Real-Time instrument with a 3-step protocol: one cycle of $8 \mathrm{~min}$ at $95^{\circ} \mathrm{C}$, then 40 cycles of $15 \mathrm{~s}$ at $95^{\circ} \mathrm{C}, 25 \mathrm{~s}$ at $60^{\circ} \mathrm{C}$, and $35 \mathrm{~s}$ at $72{ }^{\circ} \mathrm{C}$ and final polymerization at $72{ }^{\circ} \mathrm{C}$ for $10 \mathrm{~min}$. Cycles of quantification $(\mathrm{Cq})$ values were generated by the CFX Manager software. The specificity of the PCR product was confirmed by melting curve analysis.

\subsection{In Vitro Virus Spread in Infected Chicken Embryo Fibroblasts}

Chicken embryo fibroblasts (CEFs) were individually derived from minced 10-dayold embryos, as described previously [32], and cultured in a mixture of two parts of DMEM and one part of F-12 medium supplemented with 5\% calf serum (CS), 5\% FCS, $1 \%$ chicken serum, and penicillin/streptomycin $(100 \mu \mathrm{g} / \mathrm{mL}$ each) in a $5 \% \mathrm{CO} 2$ atmosphere at $37{ }^{\circ} \mathrm{C}$. The tva genotypes were determined by PCR and capillary sequencing. For PCR, we used primers TVA-fw ( $5^{\prime}$ GCATGGTGCGGTTGTTGGAG3') and TVA-rv ( $5^{\prime}$ CTGTGCCGCCGGCGGTGGGC3') with ExTaq HS DNA Polymerase (TaKaRa) and cycling conditions as follows: $98^{\circ} \mathrm{C}$ for $3 \mathrm{~min}, 40$ cycles of $98^{\circ} \mathrm{C}$ for $10 \mathrm{~s}, 65^{\circ} \mathrm{C}$ for $30 \mathrm{~s}, 72^{\circ} \mathrm{C}$ for $30 \mathrm{~s}$, and $72{ }^{\circ} \mathrm{C}$ for $5 \mathrm{~min}$ [16]. The RCASBP(A)GFP and RCASBP(K)GFP viruses transducing GFP were used for infection in $100 \mu \mathrm{L}$ of media. Virus infection and in vitro spread of the virus were quantitatively assessed as an increasing percentage of GFP-positive cells by flow cytometry on day 3 p.i.

\subsection{In Vivo Induction of Tumors with Transforming Virus Pseudotype}

The transforming virus of A or K subgroup specificity was rescued from 16Q cells [33], which contain replication-defective and v-src containing BH-RSV. 16Q cells were cocultured for 5 days with RCASBP(A)GFP- or RCASBP(K)GFP-infected DF-1 cells, and virus stocks consisting of GFP-reporter viruses and transforming viruses were harvested and frozen at $-80^{\circ} \mathrm{C}$. The titer of the transformation component was assessed as $10^{4}$ and $10^{5}$ focus-forming units (FFU)/mL for subgroups $\mathrm{A}$ and $\mathrm{K}$, respectively, by a focus assay in Brown Leghorn chicken embryo fibroblasts. $t v a+/+, t v a+/-$, and $t v a-/-$ chickens at the age of 23 to 39 days were inoculated with 500 or $1000 \mathrm{FFU}$ of transforming virus in $0.1 \mathrm{~mL}$ of culture media subcutaneously into the left and right wing web, respectively. The sarcoma induction at the site of inoculation was followed and quantitated as the weight of tumors dissected from euthanized birds.

\subsection{Quantification of MMA Serum Levels}

MMA was determined by commercial kit ClinMass ${ }^{\circledR \circledR}$ Komplettkit, advanced (RECIPE Chemicals + Instruments, GmbH, München, Germany). LC-MS/MS analysis was carried out in Agilent 1290 Infinity LC System (Agilent Technologies, Palo Alto, CA, USA) coupled with an API 4000 triple quadrupole mass spectrometer with an electrospray ion source using the positive electrospray ionization technique and selected multiple reaction monitoring. 


\section{Results}

\subsection{Introduction of Tva Frame-Shift Deletions in Chicken PGCs}

In order to generate tva $-/$ - chicken PGCs, we used the tva-specific GFP-transducing CRISPR/Cas9 vector described in [18]. The CRISPR/Cas9 cleavage site in the second exon of tva and the guide RNA sequence are shown in Figure 1A. This vector was nucleofected into the PGCs derived from blood aspiration of 14- to 17-stage $(\mathrm{H} \& \mathrm{H})$ donor male embryos. After nucleofection, we selected single-cell PGC clones and found two proliferative clones with indel mutations $5^{\prime}$ to the cleavage site. Clone No. 1 was heterozygous with insertion of one nucleotide (nt) in the first allele and deletion of $15 \mathrm{nt} 5^{\prime}$ to the cleavage site in the second allele. Clone No. 2 was homozygous with both alleles bearing a frame-shifting deletion of $10 \mathrm{nt} 5^{\prime}$ to the cleavage site in the second exon of $t v a$. The extent of indels is shown in Figure 1B. The PGC clone No. 2 was expanded and used for further work.

\subsection{Generation of Tva KO, the Tva -/- Chicken Line}

To generate knock-out chickens, we used the strategy of orthotopic transplantation of PGCs into the testes of adult recipient roosters and subsequent breeding of their offspring (Figure 1C). This original approach proved its efficiency in previous projects and provided transgenic and genetically edited chickens [26,30]. The PGC clone No. 2 was transplanted into two recipient roosters aged eight months. In order to replace the recipient's own germ line with orthotopically transplanted manipulated PGCs, roosters were sterilized by gamma irradiation in advance, and the decline of endogenous spermatogenesis and the restoration of spermatogenesis after PGC transplantation were monitored. Both recipients restored spermatogenesis 10 to 11 weeks after transplantation. Following artificial insemination, both intramagnal and intravaginal, we obtained $\mathrm{F}_{1}$ offspring with the expected $t v a+/-$ genotype. We did not find any chicken of the wt tva +/+ genotype, which demonstrated the efficiency of the sterilization procedure and high replacement rate in grafted testes. The whole data on recipient cockerels and founder tva $+/-$ heterozygotes are given in Table 1. After sexual maturation, $F_{1}$ chickens were mated to produce $F_{2}$ offspring, in which we observed segregation of $t v a+/+, t v a+/-$, and $t v a-/-$ genotypes (29:63:30, respectively) in correspondence with the expected Mendelian ratio (Pearson's $X^{2}$ test, $p<0.05$ ).

Table 1. Summarized data on the PGC recipient roosters and founder $t v a+/-$ heterozygotes.

\begin{tabular}{|c|c|c|c|}
\hline $\begin{array}{c}\text { Rooster No. } \\
\text { (No. of PGC Clone) }\end{array}$ & $\begin{array}{l}\text { Restoration of Spermatogenesis } \\
\text { (Weeks after Transplantation) }\end{array}$ & Hens Inseminated a & $\begin{array}{l}\text { Hatched Chickens } \\
\qquad(t v a+l-)^{a}\end{array}$ \\
\hline $1(2)$ & 10 & $2 / 3$ & $8\left(5 \sigma^{\pi} / 3\right.$ ㅇ $)$ \\
\hline $16(2)$ & 11 & $4 / 3$ & $6\left(4 \sigma^{\pi} / 2\right.$ 우) \\
\hline
\end{tabular}

${ }^{\text {a }}$ Intramagnal/intravaginal insemination.

\subsection{Absence of Mutations at Potential Off-Target Sites}

To assess the probability of non-specific non-tva mutations introduced into the genome by the CRISPR/Cas9 activity, we scanned the chicken genome using the CRISPOR tool and selected the most probable off-target sites. No potential off-target site with identical $20 \mathrm{nt}$ core sequence was found; seven sites with the highest cutting frequency determination (CFD) score (Supplementary Table S1 and Figure S1) contained three to four mismatches. The nucleotide sequence of these potential off-target sites was analyzed in the DNA of the tva - / - PGC clone 2, which was used for generation of the tva - / - chicken line, and the DNA isolated from the ejaculate of recipient rooster No. 16. We did not identify any mutation in selected potential off-target sites (Supplementary Figure S2), which indicates that our tva-designed CRISPR/Cas9 construct hits specifically in the tva locus.

\subsection{Tva -/-Chickens Are Resistant to Infection with ALV-A and ALV-K}

Having the TvaKO F $\mathrm{F}_{2}$ offspring segregating the wt $t v a+/+$, heterozygotes tva $+/-$, and tva $-/$ - knock-out chickens, we tested the resistance of $t v a-/-$ chickens to the infection 
with ALVs of A and K subgroup specificity. We challenged three to seven chickens of each three genotypes at the age of 23 to 29 days by intravenous (i.v.) injection of $10^{6} \mathrm{IU}$ of either RCASBP(A)GFP or RCASBP(K)GFP vectors, which combine the backbone of replicationcompetent RCAS vector [32] with the env genes of respective subgroup specificity, and quantitatively tested by reverse transcriptase PCR (qRT PCR), whether primary viremia was established in the blood sera collected repeatedly during the experiment. On days 9 and 13 after infection with RCASBP(K)GFP or RCASBP(A)GFP, respectively, all twa $+/+$ and $t v a+/$ - chickens tested positive with $10^{4}$ to $10^{5}$ viral genomes per $\mathrm{ml}$ of sera, whereas tva $-/$ - chickens and two mock-infected tva $+/-$ chickens remained negative (Figure 2). In parallel, we tested the presence of replication-competent GFP-transducing virus in the blood sera of the same cohorts of chickens. Again, no infectious virus, either of A or K subgroup, was detected in $t v a-/-$ chickens, whereas all tva $+/-$ and tva $+/+$ chickens displayed viremia, with the exception of one $t v a+/+$ chicken. Titers $10^{2}$ to $10^{4}$ of the A subgroup virus were detected 7 and 14 days post infection. The $K$ subgroup virus at titers $10^{1}$ to $10^{3}$ per $\mathrm{ml}$ was detected 7 days post infection and disappeared 14 days post infection in all chickens except one (Table 2).

\subsection{In Vitro Cultured Tva -/- Embryo Fibroblasts Do Not Support the Spread of ALV-A and $A L V-K$}

As an alternative assay demonstrating the resistance to ALV-A and ALV-K subgroups, we used the in vitro infection of chicken fibroblasts prepared individually from tva $\mathrm{KO}$ embryos of $t v a+/+$, $t v a+/-$, and $t v a-/-$ genotypes (two, two, and four embryos, respectively). We used GFP-transducing reporter viruses RCASBP(A)GFP and RACSBP(K)GFP for infection of cultured embryo fibroblasts, and the susceptibility to a given virus subgroup was estimated as the percentage of GFP-positive cells. Three days after infection with the RCASBP(A)GFP virus, we observed ca $35 \%$ of GFP-positive tva $+/+$ cells and $23 \%$ of GFP-positive tva +/ - cells, whereas tva - / - cells remained GFP-negative (Figure 3). Similarly, infection with the RCASBP(K)GFP virus resulted in $65 \%$ to $70 \%$ of GFP-positive tva $+/+$ cells, $58 \%$ of GFP-positive tva $+/-$ cells, and $0 \%$ in $t v a-/-$ cells. Although the multiplicity of infection was the same for both $\mathrm{A}$ and $\mathrm{K}$ subgroup viruses, we observed roughly two-fold more efficient virus spread in the case of RCASBP(K)GFP. This difference, however, was not observed in chicken DF-1 cells used as a positive control, where infection with RCASBP(A)GFP and RCASBP(K)GFP viruses resulted in roughly the same percentages of GFP-positive cells, $62.5 \%$ and $67 \%$, respectively. The absence of virus spread in tva $-/$ - chicken embryo fibroblasts confirms the resistance to ALV-A and ALV-K subgroups conferred by the knock-out of the chicken tva gene.
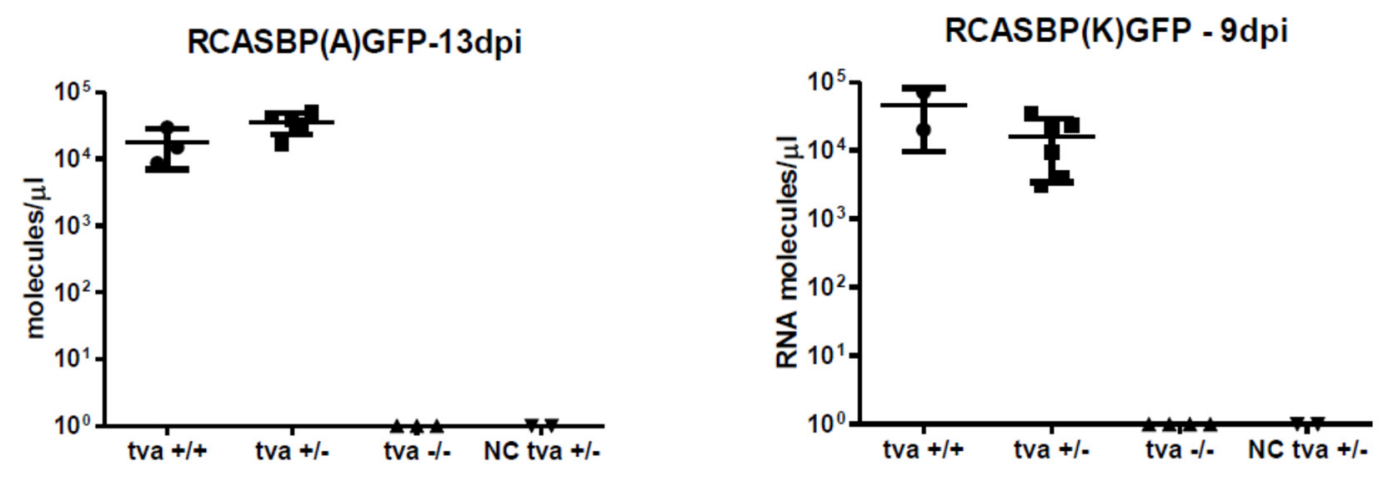

Figure 2. Resistance of $t v a \mathrm{KO}$ chickens to in vivo infection with ALV subgroup A and K. tva $+/+, t v a+/-$, and tva $-/-$ chickens were infected with either RCASBP(A)GFP (left) or RCASBP(K)GFP (right) viruses. tva +/ - chickens were also mock-injected as negative controls (NC). Primary viremia was analyzed by RT-PCR quantification of the respective env gene in the serum of RCASBP(A)GFP- or RCASBP(K)GFP-infected chickens. Each value represents the titer of viral RNA molecules as the mean of technical triplicates of individual chickens. For the groups of $t v a+/+$ and $t v a+/-$ chickens, the means \pm SD are shown. 
Table 2. Viremia in chickens inoculated with reporter ALV-A- and ALV-K-based vectors.

\begin{tabular}{cccccc}
\hline & RCASBP(A)GFP & & \multicolumn{3}{c}{ RCASBP(K)GFP } \\
\hline Genotype & Chicken No. & Virus Titer $^{\text {a }}$ & Genotype & Chicken No. & Virus Titer $^{\text {a }}$ \\
\hline$t v a-/-$ & 52 & $0 / 0$ & $t v a-/-$ & 129 & $0 / 0$ \\
$t v a-/-$ & 58 & $0 / 0$ & $t v a-/-$ & 150 & $0 / 0$ \\
$t v a-/-$ & 62 & $0 / 0$ & $t v a-/-$ & 155 & $0 / 0$ \\
$t v a+/-$ & 51 & $10^{2} / 10^{3}$ & $t v a+/-$ & 125 & $10^{3} / 0$ \\
$t v a+/-$ & 53 & $10^{4} / 10^{3}$ & $t v a+/-$ & 126 & $10^{2} / 0$ \\
$t v a+/-$ & 55 & $10^{3} / 10^{2}$ & $t v a+/-$ & 127 & $10^{1} / 0$ \\
$t v a+/-$ & 57 & $10^{2} / 10^{3}$ & $t v a+/-$ & 130 & $10^{1} / 0$ \\
$t v a+/-$ & 59 & $10^{3} / 10^{3}$ & $t v a+/-$ & 151 & $10^{2} / 0$ \\
$t v a+/+$ & 50 & $10^{2} / 10^{3}$ & $t v a+/-$ & 152 & $10^{2} / 10^{1}$ \\
$t v a+/+$ & 54 & $10^{3} / 10^{4}$ & $t v a+/-$ & 156 & $10^{2} / 0$ \\
$t v a+/+$ & 61 & $0 / 10^{2}$ & $t v a+/+$ & 128 & $10^{1} / 0$ \\
& & & $t v a+/+$ & 131 & $10^{2} / 0$ \\
& & & $t v a+/+$ & 132 & $0 / 0$ \\
& & & $t v a+/+$ & 133 & $10^{1} / 0$ \\
& & & $t v a+/+$ & 134 & $10^{1} / 0$ \\
& & & $t v a+/+$ & 148 & $10^{2} / 0$ \\
& & & & 154 & $10^{2} / 0$ \\
\hline
\end{tabular}

a $\mathrm{IU}$ per mL $7 \mathrm{dpi} / 14 \mathrm{dpi}$.
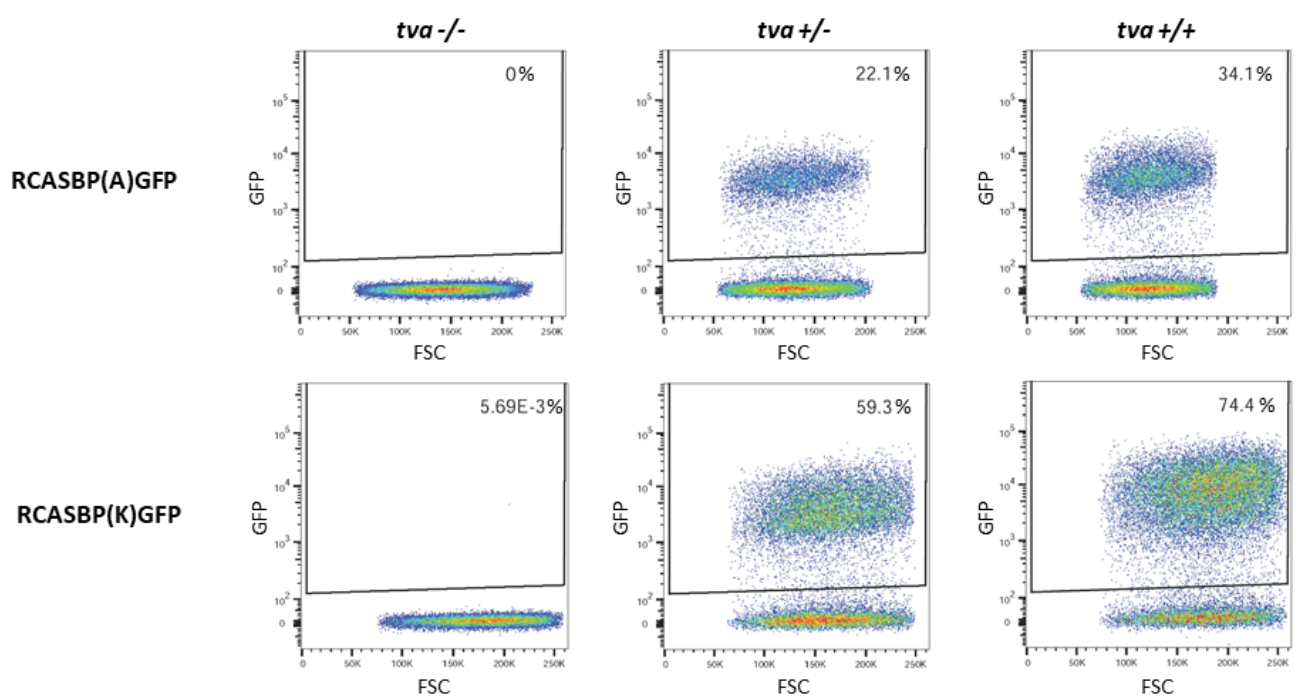

Figure 3. In vitro virus spread in embryo fibroblasts of tvaKO chickens. FACS dot-plots of tva $-/-$ (left column), $t v a+/-$ (middle column), and $t v a+/+$ chicken embryo fibroblasts infected with RCASBP(A)GFP (top row) or RCASBP(K)GFP reporter viruses. The percentages of gated GFPpositive cells are given in individual dot-plots. Representative examples of four tva $-/-$, two tva $+/-$, and two tva + + embryo fibroblast cultures are shown.

\subsection{Tva KO Chickens Are Resistant to an ASLV-A/K-Pseudotyped Transforming Virus}

Avian sarcoma and leukosis virus (ASLV) strains containing the v-src oncogene induce acute sarcomas in susceptible chickens, and the rapid growth of tumors depends on the receptor-mediated virus entry and spread. We therefore tested the virus resistance of tva $-/$ - chickens by inducing tumors with a v-src-transducing virus pseudotyped with the A or K subgroup envelope. This assay had been established in our previous ALV receptor studies for subgroups A, B, C, D, E, and J [11,30]. The titer of the transforming pseudotyped virus was determined by an in vitro focus assay as focus-forming units (FFU), and each chicken in this experiment was inoculated with $500 \mathrm{FFU}$ into one wing web and $1000 \mathrm{FFU}$ into the other wing web. Five $t v a+/+$, fifteen $t v a+/-$, and three $t v a-/-$ chickens aged 23 to 39 days were inoculated with K subgroup-pseudotyped ASLV. All tva+/ - and tva 
+/+ chickens developed rapidly growing tumors (Figure 4) 10 to 14 days after injection of both doses and had to be sacrificed 16 days p.i. In contrast, none of the three inoculated tva $-/-$ chickens developed any tumor for the next four months and remained healthy, as confirmed by autopsy. Correspondingly, seven $t v a+/+$, eleven $t v a+/-$, and six tva $-/-$ chickens aged 23 to 29 days were inoculated with A subgroup-pseudotyped ASLV, and all tva +/+ and tva+/ - chickens developed tumors 10 to 16 days after injection of both higher and lower dose and had to be sacrificed 28 days p.i. Again, all tva $-/-$ chickens remained healthy (Figure 4) and did not developed tumors for the next four months. The growth of tumors induced by A subgroup-pseudotyped ASLV was significantly slower than the growth of tumors induced by K subgroup-pseudotyped ASLV. In conclusion, these results confirm that the absence of Tva receptor confers resistance to ASLV subgroups A and K.

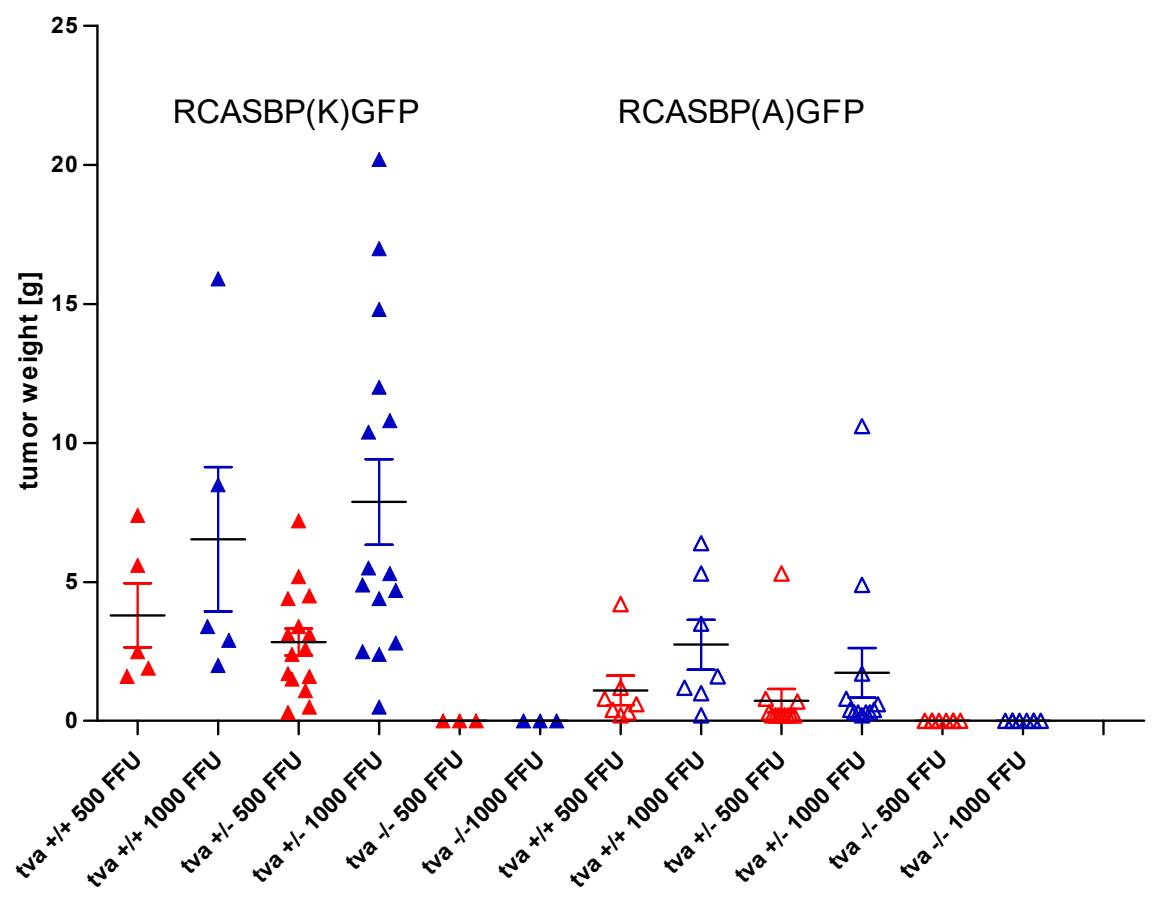

Figure 4. Resistance of tva $\mathrm{KO}$ chickens to in vivo tumor induction by transforming virus of $\mathrm{K}$ or A subgroup specificity. The $\mathrm{v}$-src transducing virus pseudotyped with either $\mathrm{K}$ subgroup RCASBP(K)GFP (left, full triangles) or A subgroup RACSBP(A)GFP (right, open triangles) was applied in lower or higher dose of $500 \mathrm{FFU}$ (red triangles) or $1000 \mathrm{FFU}$ (blue triangles) to chickens of all genotypes (groups of 3 to 15 chickens). Weights of tumors dissected 16 d.p.i. are given as individual values and means $\pm \mathrm{SD}$.

\subsection{TvaKO Chickens Have Increased Serum Levels of Methylmalonic Acid}

The physiological function of the Tva receptor was recently identified as the uptake of transcobalamin II bound cbl. In order to demonstrate that the cbl is affected in tva - / chickens, we measured the serum levels of MMA, which is the most sensitive indicator of functional cbl depletion. In five tva $-/$ - chickens, the levels of MMA reached 19 to $44 \mu \mathrm{mol} / \mathrm{L}$, which is significantly higher than 1 to $5 \mu \mathrm{mol} / \mathrm{L}$ in eight tva $+/-$ chickens and 1 to $2 \mu \mathrm{mol} / \mathrm{L}$ in five tva $+/+$ chickens (Figure 5). This finding is in accordance with our expectations, because methylmalonyl-CoA is the substrate for adenosylcobalamin-dependent conversion to succinyl-CoA, and MMA elevation in tva $-/-$ chickens is congruent with its release from the accumulating reaction substrate under conditions of inefficient cbl uptake. 


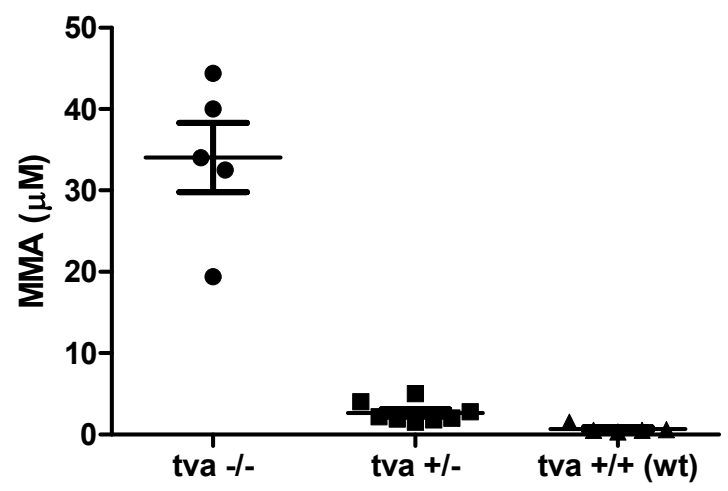

Figure 5. Serum levels of methylmalonic acid in $t v a-/-$ (circles), $t v a+/-$ (squares), and wild-type tva $+/+$ (triangles) chickens. Data are shown as serum levels ( $\mu$ mol per $\mathrm{L})$ in individual chickens and means \pm SD.

\section{Discussion}

In this study, we demonstrated that homozygous in vivo knock-out of the chicken twa gene by a frame-shifting deletion in the second exon results in complete resistance to two ALV subgroups, A and $\mathrm{K}$, which share Tva as an entry receptor. After ALV$\mathrm{J}$, where the complete resistance was achieved by gene editing of the chicken $\mathrm{Na}^{+} / \mathrm{H}^{+}$ exchanger $1[30,34]$, it is the second example of artificial anti-viral resistance conferred by genetic manipulation of the chicken. In pigs, this strategy was employed for resistance to the porcine reproductive and respiratory syndrome virus and transmissible gastroenteritis virus by editing specific receptors CD136 [35] and pAPN [36]. The genetic manipulation of virus receptors is a promising anti-viral approach that targets the virus before it penetrates the host cell. Of course, before any practical application, other studies using field strains of the virus and commercial lines of chicken will be required. Another concern is the possibility that ALV-A could adapt to the tva deletion and overcome the resistance of TvaKO line. This possibility should be tested both in vitro and in vivo before the practical application of this strategy.

In the chicken, however, this approach is hampered by the laboriousness and low efficacy of the procedures leading to the genetic knock-out or gene editing. This technology mostly relies on in vitro manipulation of PGCs, which must be reintroduced into embryos and matured to functional sperm [37]. Up to now, only a few examples of genetic knockout have been reported [38,39]. Orthotopic transplantation of PGCs into the testes of sterilized recipients [26] has proven to be an efficient procedure in production of geneedited poultry [30] and, at present, in the generation of the tva gene knock-out. Quite recently, another approach of CRISPR/Cas9 delivery into the chicken embryo blastoderm by adenoviral vector led to genetic knock-out of the melanophilin gene in quail and to the change in the plumage color [40]. Very promising and versatile resource is the Cas9 stably expressed in all tissues of transgenic animals, which facilitates in vivo organ-specific gene editing. This strategy was recently established in parallel in pigs and chickens [41]. The last addition to the PGC technology in the chicken is the system of inducible germ line ablation and introduction of donor genome-edited PGC into the surrogate host chicken [42].

In addition to its function as ALV receptor, Tva was recently identified as a specific receptor for cbl-saturated transcobalamin necessary for efficient cbl internalization into cells [21]. Thus, Tva ensures the bioavailability of cbl for internal conversions of MMACoA to succinyl-CoA and remethylation of homocysteine. Creation of the chicken tva knock-out is therefore interesting not only for the virus-resistant phenotype, but also as a potential model for the cbl metabolism. Genetic knock-out of orthologous CD320 in the mouse already exists [23], but the peculiarities of avian cbl uptake and transport, such as the absence of haptocorin (also known as transcobalamin 1) [43], and the importance of domestic poultry for human nutrition substantiate the use of a comparative chicken model. Genomic searches have shown that birds have only two cbl carrier proteins: transcobalamin 
(equivalent of mammalian transcobalamin 2) and gastric intrinsic factor [43,44], which binds $\mathrm{cbl}$ in the gastrointestinal tract and transports it to the blood. Since their separation from other vertebrate lineages, birds have lost haptocorrin, which in mammals is found in plasma, where it serves as a high affinity storage protein for $\mathrm{cbl}$ and its derivatives. Not unexpectedly, avian transcobalamin was found to display some protein motifs resembling haptocorrin and might partially substitute its role [43].

Another important cbl-dependent metabolic pathway is the conversion of homocysteine to methionine, where methyl-cbl serves as a methionine synthase cofactor necessary for the transfer of methyl groups from methyl tetrahydrofolate to homocysteine. Further conversion of methionine to S-adenosylmethionine, a universal donor of the methyl group [45], produces a critical substrate for biological methylation of proteins and nucleic acids. cbl depletion observed in the brains of CD320 KO mice was accompanied by strongly decreased global DNA methylation [46]. This suggests that blocks in the cbl uptake could affect epigenetic regulations throughout the genome and exert unexpected phenotypic changes in Tva- or CD320-depleted animals. For example, advanced cbl deficiency has been shown to contribute to nervous system demyelination and epigenetic factors such as histone modifications contribute to this phenotype [47].

The previously developed animal model of cbl depletion, the CD320 KO mouse, displayed specific neuropathology and behavioral alterations in addition to the aforementioned metabolic changes. Axonal demyelination in the spinal cord leading to partial loss of peripheral sensation [24] and the decreased brain mass and hippocampal nuclei were accompanied by anxiety and defects in learning, memory, and spatial orientation [48]. The exact phenomic analysis remains to be done in our comparative model. We did not observe any gross phenotypic effect segregating with the tva deficiency; the TvaKO chickens are normally fertile and do not display any special pathology. However, our analysis is based on a small number of outbred chickens available in the early phase of TvaKO line breeding, and slight effects on highly polymorphic traits such as growth or immunity cannot be analyzed in our system. Therefore, we will prepare an additional TvaKO line on the inbred background. Actually, the absence of specific growth retardation is true for already existing inbred chicken line $7_{2}$, bearing the frame-shifting four-nucleotide insertion in the tva gene [9], which has long been maintained in captivity without any deleterious phenotype. We, however, cannot exclude compensatory genetic changes in this case. The absence of distinct phenotype is consistent with the mouse model, where CD320 knock-out does not completely abrogate the cbl import, and clinically asymptomatic human cases with CD320 defect $[49,50]$, implying a parallel transport mechanism [23,24]. An alternative cbl uptake pathway is also visible in the tva chicken cells, where the uptake of cbl-transcobalamin remains significantly above the background level [21]. MMA is the most sensitive marker of altered cbl metabolism, whereas other metabolites will be analyzed in the inbred Tva $\mathrm{KO}$ line.

In vivo application of CRISPR/Cas9 in the livestock raises concerns of potential offtarget mutations. Therefore, we selected the most probable off-target sites and analyzed them by PCR amplification and sequencing. We concluded that our CRISPR/Cas9 targeting of the chicken tva locus was very specific with no off-target mutations introduced into the loci with the highest CFD score. The ultimate solution would be to perform wholegenome analysis, applying next-generation sequencing technology to the freshly expanded PGC clones. On the other hand, we can expect that potential off-target mutations would disappear by random drift during the subsequent breeding of $F_{1}$ and $F_{2}$ offspring.

In conclusion, we suggest that the introduction of genome-edited chicken lines could help in reducing economic losses in the poultry industry, provided that Tva depletion does not affect the production performance. More precise modification of Tva altering a single amino acid residue critical for virus binding, e.g., C40W substitution present in the tva $a^{r}$ allele [9], might be convenient for maintaining the normal production performance. On the other hand, the Tva knock-out generated by a frame-shifting deletion confirmed the cross-resistance to ALV-A and ALV-K and provided a new animal model for studies of 
cbl depletion in vivo. Although there exist naturally arisen virus-resistant tva alleles in inbred chicken lines, their introgression into commercial lines using conventional breeding would be a slow process that requires multiple rounds of backcrosses and could take many years. In the case of previously prepared resistance to ALV-J, we did not find any resistanceassociated natural polymorphism in the chicken [51], and we identified the critical amino acid, W38, by comparison of resistant and susceptible galliform species $[47,52,53]$. The gene editing technology in combination with PGC orthotopic transplantation that we demonstrated in this and previous work $[26,30]$ offers a one-generation shortcut of such lengthy process and allows for the maintenance of desirable qualities of the original line without gene penetration from the donor line.

Supplementary Materials: The following are available online at https:/ / www.mdpi.com/article/10 $.3390 /$ v13122504/s1, Figure S1. Amplified nucleotide sequences of potential off-targets loci. Positions of primers used for PCR amplification (underlined) and sequences complementary to gRNA (bold) are shown. Figure S2. Nucleotide sequences of potential off-target sites amplified from PGC tva - / clone 2 and ejaculate of recipient rooster No. 16 aligned to the NCBI chicken genome. Sequencing primers (either forward or reverse) are highlighted with green color and the gRNA complementary sequence with red color. Table S1. List of potential off-target sites with the highest CFD score and the core sequence of gRNA. The mismatches between gRNA and the off-target site are in bold, and the protospacer adjacent motifs are in italics.

Author Contributions: Conceptualization, project co-ordination, funding acquisition, and supervision, J.H. and P.T.; investigation, A.K., P.T., J.M., J.K. (Jakub Krijt), V.K. (Veronika Krchlíková), V.K. (Viktor Kožich), J.P., J.G. and D.E.; methodology, A.K., P.T., J.M., J.K. (Jakub Krijt), J.P., V.K. (Veronika Krchlíková), D.K., M.R., J.K. (Jiř́ Kalina) and F.Š.; resources, J.H., P.T., J.P. and D.E.; writing and editing: J.H., A.K., P.T., J.M., D.E. and V.K. (Viktor Kožich); funding acquisition, J.H., P.T. and V.K. (Viktor Kožich). All authors have read and agreed to the published version of the manuscript.

Funding: This work was supported by the Ministry of Agriculture of the Czech Republic (grant no. QK1810344 awarded to J.H. and P.T.) and the Czech Academy of Sciences (Premium Academiae Award to J.H.). We also acknowledge institutional support from project RVO 68378050. Viktor Kožich and Jakub Krijt were in part supported by the 19-08786S grant from the Czech Science Foundation and received institutional support from project RVO-VFN 64165. The funders had no role in the study design, data collection and analysis, decision to publish, or preparation of the manuscript.

Institutional Review Board Statement: Not applicable.

Informed Consent Statement: Not applicable.

Data Availability Statement: All data are contained within the article or Supplementary Materials.

Acknowledgments: The authors acknowledge Jitka Sokolová (Charles University, First Faculty of Medicine and General University Hospital in Prague) for help with the analysis of MMA and Martina Minariková, Anna Burgerová (both from the chicken facility of the Institute of Molecular Genetics, Prague), and Barbora Benešová (BIOPHARM) for their help with maintaining and breeding the gene-edited chicken lines.

Conflicts of Interest: The authors declare that no competing interest exist.

\section{References}

1. Federspiel, M.J. Reverse engineering provides insight on the evolution of subgroups A to E avian sarcoma and leukosis virus receptor specificity. Viruses 2019, 11, 497. [CrossRef]

2. Bates, P.; Young, J.A.; Varmus, H.E. A receptor for subgroup a Rous sarcoma virus is related to the low density lipoprotein receptor. Cell 1993, 74, 1043-1051. [CrossRef]

3. Young, J.A.T.; Bates, P.; Varmus, H.E. Isolation of a chicken gene that confers susceptibility to infection by subgroup A avian leukosis and sarcoma viruses. J. Virol. 1993, 67, 1811-1816. [CrossRef] [PubMed]

4. Brojatsch, J.; Naughton, J.; Rolls, M.M.; Zingler, K.; Young, J.A. CAR1, a TNFR-related protein, is a cellular receptor for cytopathic avian leukosis-sarcoma viruses and mediates apoptosis. Cell 1996, 87, 845-855. [CrossRef]

5. Adkins, H.B.; Brojatsch, J.; Naughton, J.; Rolls, M.M.; Pesola, J.M.; Young, J.A. Identification of a cellular receptor for subgroup E avian leukosis virus. Proc. Natl. Acad. Sci. USA 1997, 94, 11617-11622. [CrossRef] 
6. Adkins, H.B.; Brojatsch, J.; Young, J.A.T. Identification and characterization of a shared TNFR-related receptor for subgroup B, D, and $\mathrm{E}$ avian leukosis viruses reveal cysteine residues required specifically for subgroup E viral entry. J. Virol. 2000, 74, 3572-3578. [CrossRef]

7. Elleder, D.; Stepanets, V.; Melder, D.C.; Šenigl, F.; Geryk, J.; Pajer, P.; Plachý, J.; Hejnar, J.; Svoboda, J.; Federspiel, M.J. The receptor for the subgroup $C$ avian sarcoma and leukosis viruses, Tvc, is related to mammalian butyrophilins, members of the immunoglobulin superfamily. J. Virol. 2005, 79, 10408-10419. [CrossRef]

8. Chai, N.; Bates, P. Na+/H+ exchanger type 1 is a receptor for pathogenic subgroup J avian leukosis virus. Proc. Natl. Acad. Sci. USA 2006, 103, 5531-5536. [CrossRef]

9. Elleder, D.; Melder, D.C.; Trejbalova, K.; Svoboda, J.; Federspiel, M.J. Two different molecular defects in the Tva receptor gene explain the resistance of two tvar lines of chickens to infection by subgroup A avian sarcoma and leukosis viruses. J. Virol. 2004, 78, 13489-13500. [CrossRef] [PubMed]

10. Klucking, S.; Adkins, H.B.; Young, J.A.T. Resistance to infection by subgroups B, D, and E avian sarcoma and leukosis viruses is explained by a premature stop codon within a resistance allele of the tvb receptor gene. J. Virol. 2002, 76, 7918-7921. [CrossRef] [PubMed]

11. Reinišová, M.; Šenigl, F.; Yin, X.; Plachý, J.; Geryk, J.; Elleder, D.; Svoboda, J.; Federspiel, M.J.; Hejnar, J. A single-amino-acid substitution in the TvbS1 receptor results in decreased susceptibility to infection by avian sarcoma and leukosis virus subgroups $\mathrm{B}$ and $\mathrm{D}$ and resistance to infection by subgroup E in vitro and in vivo. J. Virol. 2008, 82, 2097-2105. [CrossRef] [PubMed]

12. Přikryl, D.; Plachý, J.; Kučerová, D.; Koslová, A.; Reinišová, M.; Šenigl, F.; Hejnar, J. The Novel Avian Leukosis Virus Subgroup K Shares Its Cellular Receptor with Subgroup A. J. Virol. 2019, 93, e00580-19. [CrossRef]

13. Rong, L.; Bates, P. Analysis of the subgroup a avian sarcoma and leukosis virus receptor: The 40-residue, cysteine-rich, low-density lipoprotein receptor repeat motif of tva is sufficient to mediate viral entry. J. Virol. 1995, 69, 4847-4853. [CrossRef] [PubMed]

14. Zingler, K.; Belanger, C.; Peters, R.; Agard, D.; Young, J.A.T. Identification and characterization of the viral interaction determinant of the subgroup a avian leukosis virus receptor. J. Virol. 1995, 69, 4261-4266. [CrossRef]

15. Rong, L.; Gendron, K.; Bates, P. Conversion of a human low-density lipoprotein receptor ligand-binding repeat to a virus receptor: Identification of residues important for ligand specificity. Proc. Natl. Acad. Sci. USA 1998, 95, 8467-8472. [CrossRef]

16. Reinišová, M.; Plachý, J.; Trejbalová, K.; Šenigl, F.; Kučerová, D.; Geryk, J.; Svoboda, J.; Hejnar, J. Intronic deletions that disrupt mRNA splicing of the tva receptor gene result in decreased susceptibility to infection by avian sarcoma and leukosis virus subgroup A. J. Virol. 2012, 86, 2021-2030. [CrossRef]

17. Chen, W.G.; Liu, Y.; Li, H.X.; Chang, S.; Shu, D.M.; Zhang, H.M.; Chen, F.; Xie, Q. Intronic deletions of tva receptor gene decrease the susceptibility to infection by avian sarcoma and leukosis virus subgroup A. Sci. Rep. 2015, 5, 9900. [CrossRef]

18. Koslová, A.; Kučerová, D.; Reinišová, M.; Geryk, J.; Trefil, P.; Hejnar, J. Genetic Resistance to Avian Leukosis Viruses Induced by CRISPR/Cas9 Editing of Specific Receptor Genes in Chicken Cells. Viruses 2018, 10, 605. [CrossRef]

19. Lee, H.J.; Park, K.J.; Lee, K.Y.; Yao, Y.X.; Nair, V.; Han, J.Y. Sequential disruption of ALV host receptor genes reveals no sharing of receptors between ALV subgroups A, B, and J. J. Anim. Sci. Biotechnol. 2019, 10, 23. [CrossRef] [PubMed]

20. Quadros, E.V.; Nakayama, Y.; Sequeira, J.M. The Protein and the Gene Encoding the Receptor for the Cellular Uptake of Transcobalamin-Bound Cobalamin. Blood 2009, 113, 186-192. [CrossRef]

21. Krchlíková, V.; Mikešová, J.; Geryk, J.; Bařinka, C.; Nexo, E.; Fedosov, S.N.; Kosla, J.; Kučerová, D.; Reinišová, M.; Hejnar, J.; et al. The avian retroviral receptor Tva mediates the uptake of transcobalamin 1 bound vitamin B12 (cobalamin). J. Virol. 2021, 95, e02136-20. [CrossRef] [PubMed]

22. Green, R.; Allen, L.H.; Bjørke-Monsen, A.-L.; Brito, A.; Guéant, J.-L.; Miller, J.W.; Molloy, A.M.; Nexo, E.; Stabler, S.; Toh, B.H.; et al. Vitamin B12 Deficiency. Nat. Rev. Dis. Primers 2017, 3, 17040. [CrossRef]

23. Lai, S.C.; Nakayama, Y.; Sequeira, J.M.; Wlodarczyk, B.J.; Cabrera, R.M.; Finnell, R.H.; Bottiglieri, T.; Quadros, E.V. The Transcobalamin Receptor Knockout Mouse: A Model for Vitamin B12 Deficiency in the Central Nervous System. FASEB J. 2013, 27, 2468-2475. [CrossRef]

24. Arora, K.; Sequeira, J.M.; Alarcon, J.M.; Wasek, B.; Arning, E.; Bottiglieri, T.; Quadros, E.V. Neuropathology of Vitamin B Deficiency in the Cd320 Mouse. FASEB J. 2019, 33, 2563-2573. [CrossRef] [PubMed]

25. Trefil, P.; Mičáková, A.; Mucksová, J.; Hejnar, J.; Poplštein, M.; Bakst, M.R.; Kalina, J.; Brillard, J.P. Restoration of spermatogenesis and male fertility by transplantation of dispersed testicular cells in the chicken. Biol. Reprod. 2006, 75, 575-581. [CrossRef]

26. Trefil, P.; Aumann, D.; Koslová, A.; Mucksová, J.; Benešová, B.; Kalina, J.; Wurmser, C.; Fries, R.; Elleder, D.; Schusser, B.; et al. Male fertility restored by transplanting primordial germ cells into testes: A new way towards efficient transgenesis in chicken. Sci. Rep. 2017, 7, 14246. [CrossRef]

27. Plachý, J. The chicken-A laboratory animal of the class Aves. Folia Biol. 2000, 46, 17-23.

28. Mucksová, J.; Reinišová, M.; Kalina, J.; Lejčková, B.; Hejnar, J.; Trefil, P. Conservation of chicken male germline by orthotopic transplantation of primordial germ cells from genetically distant donors. Biol. Reprod. 2019, 101, 200-207. [CrossRef]

29. Whyte, J.; Glover, J.D.; Woodcock, M.; Brzeszczynska, J.; Taylor, L.; Sherman, A.; Kaiser, P.; McGrew, M.J. FGF, insulin, and SMAD signaling cooperate for avian primordial germ cell self-renewal. Stem Cell Rep. 2015, 5, 1171-1182. [CrossRef]

30. Koslová, A.; Trefil, P.; Mucksová, J.; Reinišová, M.; Plachý, J.; Kalina, J.; Kučerová, D.; Geryk, J.; Krchlíková, V.; Lejčková, B.; et al. Precise CRISPR/Cas9 editing of the NHE1 gene renders chickens resistant to the J subgroup of avian leukosis virus. Proc. Natl. Acad. Sci. USA 2020, 117, 2108-2112. [CrossRef] 
31. Ran, F.A.; Hsu, P.D.; Wright, J.; Agarwala, V.; Scott, D.A.; Zhang, F. Genome engineering using the CRISPR-Cas9 system. Nat. Protoc. 2013, 8, 2281-2308. [CrossRef]

32. Federspiel, M.J.; Hughes, S.H. Retroviral gene delivery. Methods Cell Biol. 1997, 52, 179-214.

33. Moscovici, C.; Moscovici, M.G.; Jimenez, H.; Lai, M.M.; Hayman, M.J.; Vogt, P.K. Continuous tissue culture cell lines derived from chemically induced tumors of Japanese quail. Cell 1977, 11, 95-103. [CrossRef]

34. Kheimar, A.; Klinger, R.; Bertzbach, L.D.; Sid, H.; Yu, Y.; Conradie, A.M.; Schade, B.; Böhm, B.; Preisinger, R.; Nair, V.; et al. A Genetically Engineered Commercial Chicken Line Is Resistant to Highly Pathogenic Avian Leukosis Virus Subgroup J. Microorganisms 2021, 9, 1066. [CrossRef]

35. Whitworth, K.M.; Rowland, R.R.; Ewen, C.L.; Trible, B.R.; Kerrigan, M.A.; Cino-Ozuna, A.G.; Samuel, M.S.; Lightner, J.E.; McLaren, D.G.; Mileham, A.J.; et al. Gene-edited pigs are protected from porcine reproductive and respiratory syndrome virus. Nat. Biotechnol. 2016, 34, 20-22. [CrossRef]

36. Xu, K.; Zhou, Y.; Mu, Y.; Liu, Z.; Hou, S.; Xiong, Y.; Fang, L.; Ge, C.; Wei, Y.; Zhang, X.; et al. CD163 and pAPN double-knockout pigs are resistant to PRRSV and TGEV and exhibit decreased susceptibility to PDCoV while maintaining normal production performance. Elife 2020, 9, e57132. [CrossRef]

37. van de Lavoir, M.C.; Diamond, J.H.; Leighton, P.A.; Mather-Love, C.; Heyer, B.S.; Bradshaw, R.; Kerchner, A.; Hooi, L.T.; Gessaro, T.M.; Swanberg, S.E.; et al. Germline transmission of genetically modified primordial germ cells. Nature 2006, 441, 766-769. [CrossRef]

38. Schusser, B.; Collarini, E.J.; Yi, H.; Izquierdo, S.M.; Fesler, J.; Pedersen, D.; Klasing, K.C.; Kaspers, B.; Harriman, W.D.; van de Lavoir, M.C.; et al. Immunoglobulin knockout chickens via efficient homologous recombination in primordial germ cells. Proc. Natl. Acad. Sci. USA 2013, 110, 20170-20175. [CrossRef]

39. Kim, G.D.; Lee, J.H.; Song, S.; Kim, S.W.; Han, J.S.; Shin, S.P.; Park, B.C.; Park, T.S. Generation of myostatin-knockout chickens mediated by D10A-Cas9 nickase. FASEB J. 2020, 34, 5688-5696. [CrossRef]

40. Lee, J.; Ma, J.; Lee, K. Direct delivery of adenoviral CRISPR/Cas9 vector into the blastoderm for generation of targeted gene knockout in quail. Proc. Natl. Acad. Sci. USA 2019, 116, 13288-13292. [CrossRef]

41. Rieblinger, B.; Sid, H.; Duda, D.; Bozoglu, T.; Klinger, R.; Schlickenrieder, A.; Lengyel, K.; Flisikowski, K.; Flisikowska, T.; Simm, N.; et al. Cas9-expressing chickens and pigs as resources for genome editing in livestock. Proc. Natl. Acad. Sci. USA 2021, 118, e2022562118. [CrossRef]

42. Ballantyne, M.; Woodcock, M.; Doddamani, D.; Hu, T.; Taylor, L.; Hawken, R.J.; McGrew, M.J. Direct allele introgression into pure chicken breeds using Sire Dam Surrogate (SDS) mating. Nat. Commun. 2021, 12, 659. [CrossRef]

43. Lopes-Marques, M.; Ruivo, R.; Delgado, I.; Wilson, J.M.; Aluru, N.; Castro, L.F.C. Basal gnathostomes provide unique insights into the evolution of vitamin B12 binders. Genome Biol. 2015, 7, 457-464. [CrossRef]

44. Greibe, E.; Fedosov, S.; Nexo, E. The cobalamin-binding protein in zebrafish is an intermediate between the three cobalaminbinding proteins in human. PLoS ONE 2012, 7, 35660. [CrossRef]

45. Selhub, J. Folate, vitamin B12 and vitamin B6 and one carbon metabolism. J. Nutr. Health Aging 2002, 6, 39-42.

46. Fernàndez-Roig, S.; Lai, S.-C.; Murphy, M.M.; Fernandez-Ballart, J.; Quadros, E.V. Vitamin B12 deficiency in the brain leads to DNA hypomethylation in the TCblR/CD320 knockout mouse. Nutr. Metab. 2012, 9, 41. [CrossRef]

47. Shen, S.; Li, J.; Casaccia-Bonnefil, P. Histone modifications affect timing of oligodendrocyte progenitor differentiation in the developing rat brain. J. Cell Biol. 2005, 169, 577-589. [CrossRef]

48. Arora, K.; Sequeira, J.M.; Hernández, A.I.; Alarcon, J.M.; Quadros, E.V. Behavioral alterations are associated with vitamin B12 deficiency in the transcobalamin receptor/CD320 KO mouse. PLoS ONE 2017, 12, e0177156. [CrossRef]

49. Quadros, E.V.; Lai, S.C.; Nakayama, Y.; Sequeira, J.M.; Hannibal, L.; Wang, S.; Jacobsen, D.W.; Fedosov, S.; Wright, E.; Gallagher, R.C.; et al. Positive newborn screen for methylmalonic aciduria identifies the first mutation in TCblR/CD320, the gene for cellular uptake of transcobalamin-bound vitamin B(12). Hum. Mutat. 2010, 31, 924-929. [CrossRef]

50. Hannah-Shmouni, F.; Cruz, V.; Schulze, A.; Mercimek-Andrews, S. Transcobalamin receptor defect: Identification of two new cases through positive newborn screening for propionic/methylmalonic aciduria and long-term outcome. Am. J. Med. Genet. A 2018, 176, 1411-1415. [CrossRef]

51. Reinišová, M.; Plachý, J.; Kučerová, D.; Šenigl, F.; Vinkler, M.; Hejnar, J. Genetic diversity of NHE1, receptor for subgroup J avian leukosis virus, in domestic chicken and wild anseriform species. PLoS ONE 2016, 11, e0150589. [CrossRef]

52. Kučerová, D.; Plachý, J.; Reinišová, M.; Šenigl, F.; Trejbalová, K.; Geryk, J.; Hejnar, J. Nonconserved tryptophan 38 of the cell surface receptor for subgroup J avian leukosis virus discriminates sensitive from resistant avian species. J. Virol. 2013, 87, 8399-8407. [CrossRef]

53. Plachý, J.; Reinišová, M.; Kučerová, D.; Šenigl, F.; Stepanets, V.; Hron, T.; Trejbalová, K.; Elleder, D.; Hejnar, J. Identification of New World quails susceptible to infection with avian leukosis virus subgroup J. J. Virol. 2017, 91, e02002-16.s. [CrossRef] 